\title{
Novel Synthetic Method for Magnetic Porous Carbon Material for Efficient Adsorption of Organic Pollutants from Aqueous Solution
}

\author{
Jia Xu ${ }^{1,2}$, Junjie Chen ${ }^{1}$, Mudasir Ahmad ${ }^{1}$, Qiuyu Zhang 1,3, Baoliang Zhang 1,4,* \\ ${ }^{1}$ School of Natural and Applied Sciences Northwestern Polytechnical University, Xi'an, 710072 , \\ China. \\ ${ }^{2}$ Xi'an Key Laboratory of Functional Organic Porous Materials, Xi'an, 710129, China. \\ ${ }^{3}$ MOE Key Laboratory of Material Physics and Chemistry under Extraordinary Condition, Ministry \\ of Education, Northwestern Polytechnical University, Xi’an, 710129, China. \\ ${ }^{4}$ Sunresins New Materials Co. Ltd., Xi’an, 710072, China.
}

* Corresponding author tel: +86-029-88431675; fax: +86-029-88431675; Northwestern Polytechnical University, Youyi Road 127\#, Xi’an (710072), China. Email: blzhang@nwpu.edu.cn 

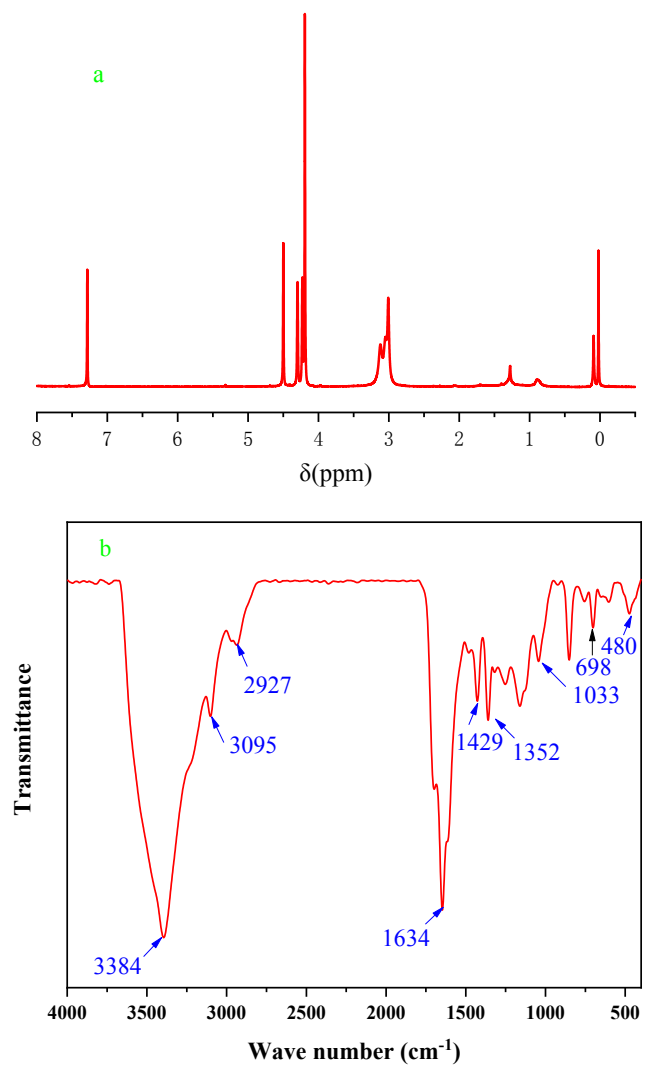

Figure $\mathrm{S} 1{ }^{1} \mathrm{H}-\mathrm{NMR}$ spectrum (a) and FTIR spectra (b) of $\mathrm{FcCH}_{2} \mathrm{Cl}$
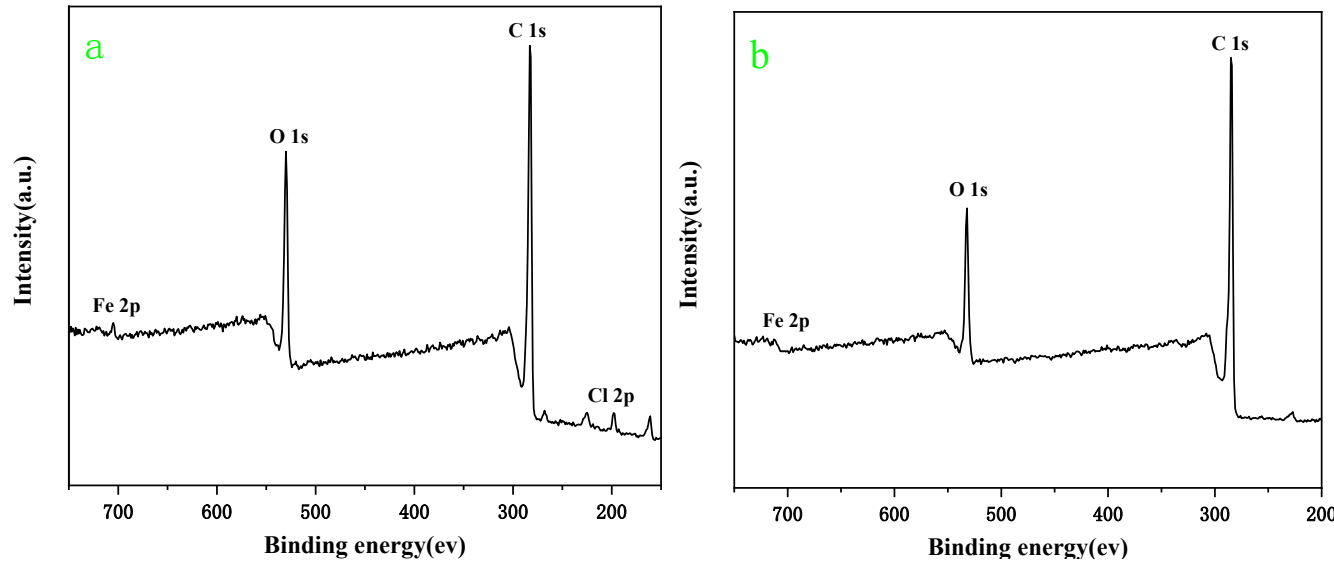

Figure S2. XPS patterns of HCFc (a) and MPMs (b) 


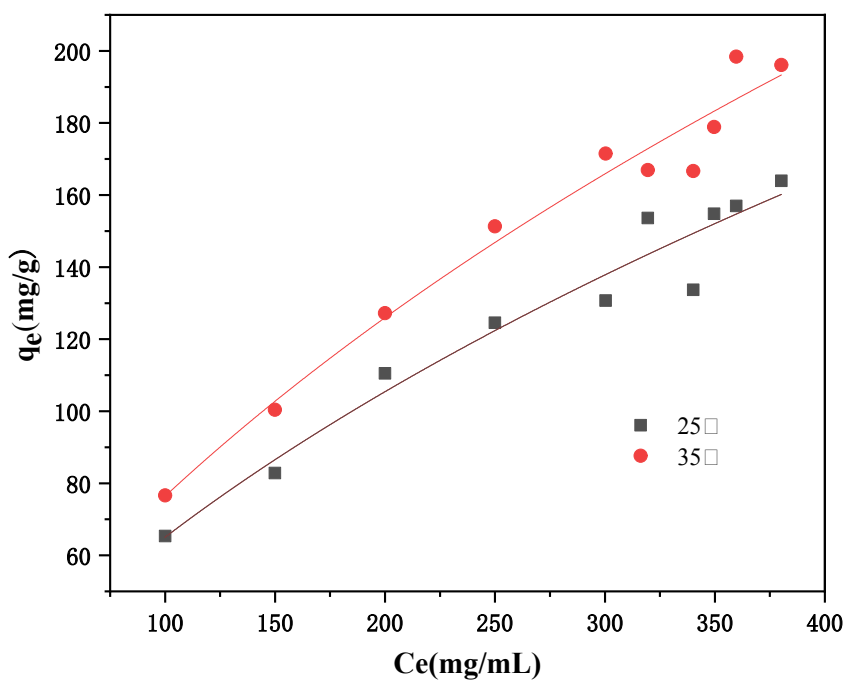

Figure S3. Nonlinear form of the Langmuir isotherm models 